\author{
JORGE J. GUMUCIO, EDITOR \\ Department of Internal Medicine \\ Division of Gastroenterology (111D) \\ VA Medical Center/University of Michigan \\ Ann Arbor, Michigan 48105
}

\section{CONSTIPATION: IS IT A RISK FACTOR FOR CHOLESTEROL GALLSTONES?}

Heaton KW, Emmett PM, Symes CL, Braddon FEM. An explanation for gallstones in normal-weight women: slow intestinal transit. Lancet 1993;341:8-10.

\section{ABSTRACT}

Many cases of gallstones can be explained in terms of the established risk factors, especially obesity. However, gallstones develop in some women who are not obese, and the causes are unknown. Biochemical studies have shown that slow intestinal transit is associated with lithogenic bile. We have tested the hypothesis that intestinal transit is abnormally slow in normal-weight women with gallstones.

In a population survey, 1058 women aged $25-69$ years, registered with general practitioners in Bristol, UK, underwent cholecystosonography. Gallstones were identified in 48 women, of whom 15 were of normal weight (body mass index $\leq 25 \mathrm{~kg} / \mathrm{m}^{2}$ ). These women and age-matched controls with healthy gallbladders then underwent measurement of whole-gut transit time (WGTT); the measurement was done directly when possible, or calculated from records of three defaecations. The mean WGTT was significantly longer in the women with gallstones than in the controls (82 vs $63 \mathrm{~h}$; mean difference $19,95 \%$ Cl $(2-37 \mathrm{~h})$. Stool output was also lower in the women with gallstones ( 74 [SD 54] vs 141 [56] g per $24 h, p=0.015)$. There was no significant difference between cases and controls in body mass index, waist-hip circumference ratio, parity, plasma triglyceride concentration, or alcohol intake.

Normal-weight women with gallstones tend to have slow intestinal transit and this feature could explain why they have gallstones.

\section{COMMENTS}

Concepts of the pathogenesis of cholesterol gallstone formation have changed over the past decade, and several defects in both the hepatic secretion of lipids and gallbladder function have been identified. We know that for crystallization of cholesterol monohydrate to occur, bile must be supersaturated with cholesterol and a gallbladder nucleating factor accelerating crystallization is required. This process may be enhanced by gallbladder hypomotility (1). Although many of non-gallstoneforming patients have thermodynamically unstable bile, it is recognized $a$ priori that bile is unstable in all gallstone-forming individuals. Supersaturated bile can

HePATOLOGY 1993;18:457-463

31/8/48389 occur as a result of an absolute increase in cholesterol secretion or a relative increase in the proportion of cholesterol to bile salt excretion. Oversecretion of cholesterol is considered the main metabolic event in women; the major cause of this defect is obesity (2). Hypersecretion of cholesterol in obese women may be compounded by weight-reduction programs that cause further increases in supersaturation of bile with cholesterol (3).

What, then, is the cause of gallstone formation in non-obese women? Possible explanations include reduced or altered bile acid pool, delayed gallbladder emptying and gallbladder factors that accelerate nucleation (1). In this study the authors attempted to determine whether prolongation of whole-gut transit time (WGTT) could be another explanation for cholesterol gallstone formation in non-obese women. To study this question, these investigators conducted a population survey of 1,058 women. Fifteen of the 48 patients with asymptomatic gallstones were of normal weight. When WGTT was examined with a radioopaque-marker technique or estimates of WGTT were made with formulas devised by the authors (4), there appeared to be a significant prolongation in transit time in gallstone patients compared with age- and weight-matched controls. Stool weights were also significantly lower in the gallstone patients; this may have reflected delayed in gut transit. The authors concluded that prolongation of WGTT would increase the percentage of deoxycholate (DCA) in the bile acid pool, favoring gallstone formation. Although this premise is attractive, several methodological problems with the study necessitate further study. First, the authors found significant overlap in WGTT: More than 50\% of non-gallstone-forming subjects had WGTT values in the range of the gallstone patients. If the subjects whose WGTT's were estimated were excluded, no major difference could be discerned between the two groups. Also, none of the patients had an extensive history of dietary factors that in themselves could alter lipid and bile acid absorption independently of WGTT. Finally, we do not know whether colonic transit time, which may prove more important, was slowed in non-obese gallstone subjects. Despite these methodological problems, the concept is intriguing; other studies suggest that alterations in colonic transit time may affect the DCA pool size and cholesterol saturation index (CSI) (5).

For instance, delays in colonic transit utilizing agents that reduce colonic motility enhance the proportion of DCA in the bile acid pool and increase CSI (5). Also, feeding DCA or cholic acid, the precursor of DCA, increases CSI (5). Conversely, in patients treated with 
laxatives, wheat bran and intestinal antibiotics, the deoxycholate pool is contracted and CSI falls $(4,5)$. Thus maneuvers that alter colonic transit affect the DCA pool size and CSI. The observation that women of childbearing age also have prolonged transit times and greater tendency toward constipation is intriguing in light of the observations that this population group is at high risk for cholesterol gallstones (6).

An increased percentage of DCA in the bile acid pool has been observed in several studies examining gallstone subjects (5). The mechanism whereby DCA increases CSI is not entirely certain, although studies indicate that excretion of this bile acid causes a greater increase in cholesterol output than does an increase in chenodeoxycholic acid (CDCA). This difference is explained by the fact that DCA is more hydrophobic; this quality may cause a greater solubilization of bile canalicular cholesterol. An additional mechanism whereby DCA would increase CSI is that an increase in DCA pool size is generally paralleled by a decrease in the CDCA pool (5). Because CDCA has the strongest inhibitory effect on cholesterol output, a change in the ratio of DCA to CDCA may have significant effects on CSI. In addition to these mechanisms, there appears to be a direct correlation between the rate of biliary excretion of DCA and arachidonic acid content of human biliary lecithin (1). Increases in this lipid fraction might enhance prostaglandin synthesis by the gallbladder mucosa. Increased prostaglandin levels would increase mucus secretion by the gallbladder epithelium, thereby providing a more favorable framework for cholesterol nucleation (1). Thus colonic factors that increase DCA pool size may enhance cholesterol gallstone formation by several mechanisms.

Should we now add prolonged WGTT to the wellknown risk factors for cholesterol gallstones (i.e., obesity, female sex, weight-reduction diets, hypertriglyceridemia and estrogens)? Certainly at this point it is difficult to do so. Krevsky et al. (7), using colonic transit scintigraphy, noted a lack of correlation between the number of bowel movements and the movement of feces through the colon. They suggest that studies counting the number of bowel movements or quantitating markers may not accurately reflect colonic transit. Improved methodologies that allow investigation to correlate colonic transit time with biliary bile acid and lipid composition would help answer this question.

JAMES L. WATKINS

THELMA E. WILEY

THOMAS J. LAYDEN

Section of Digestive and Liver Diseases

University of Illinois at Chicago

Chicago, Illinois 60612

\section{REFERENCES}

1. Hay DW, Carey MC. Pathophysiology and pathogenesis of cholesterol gallstone formation. Semin Liver Dis 1990;10:159-170.

2. Maglure KM, Hayes KC, Colditz GA, Stampfer MJ, Speizer FE, Willett WC. Weight, diet, and the risk of symptomatic gallstones in middle-aged women. N Engl J Med 1989;321:563-568.
3. Liddle RA, Goldstein RB, Saxton J. Gallstone formation during weight-reduction dieting. Arch Intern Med 1989;149:1750-1753.

4. Marcus SN, Heaton KW. Intestinal transit, deoxycholic acid and the cholesterol saturation of bile: three inter-related factors. Gut $1986 ; 27: 550-558$.

5. Marcus SN, Heaton KW. Deoxycholic acid and the pathogenesis of gallstones. Gut 1988;29:522-533.

6. Heaton KW, Radvan J, Cripps H, Mountford RA, Braddon FEM, Hughes AO. Defecation frequency and timing, and stool form in the general population: a prospective study. Gut 1992;33:818-824.

7. Krevsky B, Malmud LS, D'Ercole F, Maurer AH, Fisher RS. Colonic transit scintigraphy: a physiologic approach to the quantitative measurement of colonic transit in humans. Gastroenterology 1986;91:1102-1112.

\section{HEPATITIS B VACCINE: SHOULD WE GIVE IT A SHOT?}

Bloom BS, Hillman AL, Fendrick AM, Schwartz JS. A reappraisal of hepatitis $B$ virus vaccination strategies using cost-effectiveness analysis. Ann Intern Med 1993; 118:298-306.

Schaffner W, Gardner P, Gross PA. Hepatitis B immunization strategies: expanding the target. Ann Intern Med 1993;118:308-309.

\section{ABSTRACT}

Objective: To determine clinical and economic consequences of alternative vaccination strategies for preventing hepatitis B virus infection (HBV).

Methods: Decision analysis was used to evaluate costs, outcomes, and cost-effectiveness of three HBV management strategies ("no vaccination," "universal vaccination," and "screen and vaccinate") in four populations (newborns, 10-year-old adolescents, a high-risk adult population, and the general adult U.S. population). Information on HBV incidence and prevalence, clinical course, and management of acute illness and chronic sequelae was obtained from the literature and a panel of experts. Actual payments (costs) were obtained from Blue Cross/Blue Shield and local pharmacies. Incremental cost-effectiveness was calculated from the perspective of the payer of medical care and subjected to sensitivity analysis.

Results: Vaccination (with or without screening) prevents more disease at somewhat increased cost than no vaccination for the neonatal, adolescent, and adult populations. Vaccination (with or without screening) is a dominant strategy in adult high-risk populations (lower cost and greater benefit than no vaccination). Optimal cost-effectiveness, with nonmonetary benefits not discounted, results if all pregnant women are screened for active HBV infection, and $H B V$ vaccine and hepatitis $B$ immune globulin are administered to babies born to mothers with positive screening tests. Then HBV vaccine is administered to all children at age 10 and again 10 years later (incremental cost-per-year-of-life-saved relative to the "no vaccination" strategy is \$375). A strategy of universal newborn vaccination alone leads to an incremental cost-per-year-of-life saved of $\$ 3332$. If adolescents are vaccinated at age 10 , incremental

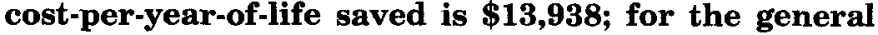
adult population, the incremental cost-per-year-of-life saved of universal vaccination is $\mathbf{\$ 5 4 , 5 2 4}$. Discounting benefits will increase cost-per-year-of-life saved 7 to 12 times for all strategies.

Conelusions: HBV vaccine is most cost-effective 